\title{
Comparison of seismic performance of strengthened historical masonry buildings under different structural designs
}

\author{
O. Isler \& V. Oztas \\ Department of Theory of Structure, Istanbul Technical University, Turkey
}

\begin{abstract}
The subject of this study is the investigation of historical masonry buildings under earthquake loads. During any earthquake the behaviour of the structural system of masonry buildings is very complicated, and the shear resistances are very low. The masonry building's wall materials, thickness, height and workmanship have a significant affect on the stability and shear resistance of the buildings. The dimensions of wall openings for windows and doors and their placing in the wall, and continuous walls or non continuous walls are also important for stability. In order to prepare a weak masonry building for a possible and heavily damaging earthquake, the structural system needs strengthening to increase its seismic performance. In this study, in order to strengthen masonry buildings, two different strengthening structural models are considered. Firstly, the masonry building is strengthened with the additional RC shear walls, secondly; the walls of the masonry building are strengthened with the FRP/GFRP grid bonded. For example, an existing and historical masonry building chosen from Istanbul is considered. Their structural systems, with strengthened and non strengthened examples, are analyzed under earthquake loads. The results of the analyses are investigated and the obtained seismic performances of the different strengthened structural systems are compared between each other. Consequently, seismic performance and lateral displacements are improved by strengthening with additional RC shear walls and/or FRP/GFRP grid bonded systems. The analyses also show the displacements of the strengthened systems are reduced and these are improved 9 and 4 times according to the present building, respectively. Finally, to maintain
\end{abstract}


outdoor views and for easy application, the FRP/GFRP grid bonded strengthened systems are recommended instead of the additional RC shear wall system. Keywords: masonry building, earthquake, strengthen.

\section{Introduction}

Unreinforced masonry (URM) walls in masonry structures have architectural and statical properties. URM walls constitute volume, protect structures against external effect and divide structure into compartments [1]. The versatility of URM walls is an advantage according to use and construction of masonry structures. In masonry structures, damage may occur because of earthquake forces, but various strengthening methods are used in order to renovate these damaged structures.

In this study, in order to strengthen masonry buildings, two different strengthening structural models and existing models are considered. Firstly, the existing model is analyzed. Secondly, the masonry building is strengthened with the additional RC shear walls. Finally, the walls of the masonry building are strengthened with the GFRP.

GFRP is composed of carbon, aramid or glass fibers with epoxy resin. GFRP has high durability, high tensile strength, resists against fatigue, and is applicable in various forms.

\section{Analyzing of a historical masonry building}

In this study, a three-story historical masonry building is analyzed with Sta4-CAD software. The dimensions of the structure are $18.11 \mathrm{~m} \times 17.10 \mathrm{~m}$ and URM wall thicknesses $33 \mathrm{~cm}$ at the basement, $22 \mathrm{~cm}$ at the other stories. This structure is in the first earthquake zone and other parameters are shown in the following table.

Table 1: $\quad$ Parameters of structure.

\begin{tabular}{|c|c|}
\hline Building Properties & Value \\
\hline \hline Soil group & $\mathrm{Z} 3$ \\
\hline Number of flat & 3 \\
\hline $\begin{array}{c}\text { earthquake acceleration } \\
\text { coefficient (Ao) }\end{array}$ & 0,4 \\
\hline Structure type coefficient $(\mathrm{R})$ & 2 \\
\hline Soil periods.(Ta/Tb) & $0,15 / 0,6$ \\
\hline Live load coefficient & 0.6 \\
\hline ground safety stress $\left(\mathrm{t} / \mathrm{m}^{2}\right)$ & 15.0 \\
\hline ground bearing coefficient $\left(\mathrm{t} / \mathrm{m}^{3}\right)$ & 1500.0 \\
\hline Gravity of concrete $\left(\mathrm{t} / \mathrm{m}^{3}\right)$ & 2,5 \\
\hline Earthquake code of Turkey [2] & TDY2007 \\
\hline
\end{tabular}



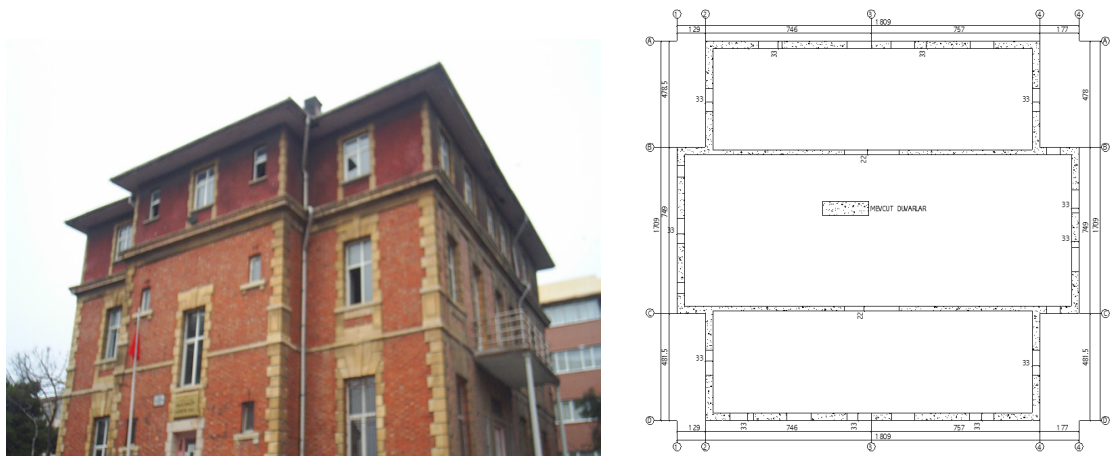

Figure 1: Examined historical building and plan.

\subsection{Analysis of existing structure}

Firstly, the existing structural system is analyzed with software and the results are examined.

The spectrum coefficient for masonry structures $\mathrm{S}(\mathrm{T})=2.5$ and earthquake load reduction coefficient for masonry system is $\mathrm{Ra}(\mathrm{t})=2$.

Earthquake loads for $\mathrm{x}$ and $\mathrm{y}$ direction are found.

$$
\begin{array}{ll}
\mathrm{Vtx}=W . A(t) / \operatorname{Ra}(\mathrm{t})>0,10 . \text { Ao.I.W } & 2259.01>180.72 \mathrm{t} \\
\mathrm{Vty}=W . A(t) / \operatorname{Ra}(\mathrm{t})>0,10 . \text { Ao.I.W } & 2259.01>180.72 \mathrm{t}
\end{array}
$$

Modal analysis minimum load ratio multiplies with equivalent earthquake loads.

Maximum earthquake load is selected from multiplicand value and modal analysis value.

$\mathrm{X}$ direction earthquake load selection:

$$
0.90 \times 2259.012=2033.111>1487.672 \rightarrow 2033.111 \mathrm{t} \quad(4.5 \mathrm{a})
$$

$\mathrm{Y}$ direction earthquake load selection:

\begin{tabular}{|c|c|c|c|c|c|c|c|c|}
\hline \multirow{2}{*}{$\begin{array}{l}\text { Kat } \\
\text { (dyf) }\end{array}$} & \multicolumn{2}{|c|}{ 9. yükleme } & \multicolumn{2}{|c|}{ 10. yükleme } & \multicolumn{2}{|c|}{ 11. yưkleme } & \multicolumn{2}{|c|}{ 12. yükleme } \\
\hline & $\delta x \quad(m)$ & $\theta z$ (rad) & $\delta x(m)$ & $\theta z \quad(r a d)$ & $\delta y \quad(m)$ & $\theta z \quad(r a d)$ & $\delta y \quad(m)$ & $\theta z$ (rad) \\
\hline 4 & $0.1487000 \mid$ & 0.0000026 & 0.1487000 & 0.0000026 & -0.258344 & -0.000106 & -0.258344 & -0.000106 \\
\hline 3 & $|0.0988657|$ & 0.0000021 & 0.0988657 & 0.0000021 & -0.183651 & -0.000057 & -0.183651 & -0.000057 \\
\hline 2 & $|0.0456890|$ & 0.0000012 & $|0.0456890|$ & $|0.0000012|$ & $\mid-0.088422$ & -0.000020 & $\mid-0.088422$ & -0.000020 \\
\hline 1 & $|0.0033021|$ & 0.0000001 & 0.0033021 & $|0.0000001|$ & -0.006277 & -0.000001 & $\mid-0.006277$ & -0.000001 \\
\hline
\end{tabular}

$$
0.90 \times 2259.012=2033.111>1533.656 \rightarrow 2033.111 \mathrm{t} \quad(4.5 \mathrm{~b})
$$

Table 2: $\quad$ Maximum displacements (cm).

Earthquake displacements are found.

Maximum displacements for existing structure are:

$$
\delta \mathrm{x}=0.148700, \delta \mathrm{y}=0.258344 \mathrm{~m} \text {. }
$$




\subsection{Analysis of masonry structure strengthened with reinforced concrete shear wall}

$30 \mathrm{~cm}$ RC shear walls are entered to the system at the $\mathrm{x}$ and $\mathrm{y}$ directions. St 420 steel bars and C30 RC are used at the strengthened system.

$$
\begin{gathered}
\text { For } \mathrm{C} 30, \mathrm{E}=318.000 \mathrm{~kg} / \mathrm{cm}^{2} \mathrm{fu}=300 \mathrm{~kg} / \mathrm{cm}^{2} \\
\text { St420 tensile stress }=4200 \mathrm{~kg} / \mathrm{cm}^{2}
\end{gathered}
$$

Equivalent RC cross-section and modulus of elasticity of RC and masonry shear wall which are worked together are found from breaking load of experiment result which are made by Franklin, S., Lynch J., Abrams D, (Performance of Rehabilitated URM Shear Walls: Flexural Behaviour of Piers) [3]

Breaking load of existing masonry wall $=\mathrm{FT}=29 \mathrm{kN}$

Breaking load of $\mathrm{RC}$ strengthened masonry wall $=\mathrm{FB}=68 \mathrm{kN}$

$$
\begin{gathered}
\mathrm{k} 1=\mathrm{FB} / \mathrm{FT}=68 / 29=2.34 \\
\text { Eeş1 }=\mathrm{ET}+\mathrm{EB} / 2.34=3000+318000 / 2.34=138897 \mathrm{~kg} / \mathrm{cm}^{2} \\
\text { beş1 }=\mathrm{bT}+\mathrm{bB} / 2.34=33+30 / 2.34=45 \mathrm{~cm}
\end{gathered}
$$

$\mathrm{RC}$ strengthened structural system is analyzed using equivalent RC crosssection and elasticity coefficient and examined results

Spectrum coefficient for strengthened masonry structures $\mathrm{S}(\mathrm{T})=2.5$ and earthquake load reduction coefficient for strengthened masonry system $\operatorname{Ra}(\mathrm{t})=2$

Earthquake loads for $\mathrm{x}$ and $\mathrm{y}$ direction are found

$$
\begin{aligned}
& \mathrm{Vtx}=\mathrm{W} . \mathrm{A}(\mathrm{t}) / \mathrm{Ra}(\mathrm{t})>0 \text {,10.Ao.I.W } 2694.02>215.52 \\
& \mathrm{Vty}=\mathrm{W} . \mathrm{A}(\mathrm{t}) / \mathrm{Ra}(\mathrm{t})>0 \text { 0,10.Ao.I.W } 2694.02>215.52
\end{aligned}
$$

Modal analysis minimum load ratio multiplies with equivalent earthquake loads.

Earthquake load select from big one between multiplicand value and modal

\begin{tabular}{|c|c|c|c|c|c|c|c|c|}
\hline \multirow{2}{*}{$\begin{array}{l}\text { Kat } \\
\text { (dyf) }\end{array}$} & \multicolumn{2}{|c|}{ 9. yükleme } & \multicolumn{2}{|c|}{ 10. yükleme } & \multicolumn{2}{|c|}{ 11. yükleme } & \multicolumn{2}{|c|}{ 12. yükleme } \\
\hline & $\delta x \quad(m)$ & $\theta z \quad(\mathrm{rad})$ & $\delta \mathrm{x} \quad(\mathrm{m})$ & $\theta z \quad($ rad) & $\delta \mathrm{y} \quad(\mathrm{m})$ & $\theta z \quad(\mathrm{rad})$ & $\delta_{\mathrm{y}} \quad(\mathrm{m})$ & $\theta z \quad(\mathrm{rad})$ \\
\hline 4 & 0.0383761 & 0.0002163 & 0.0383761 & 0.0002163 & -0.028532 & 0.0004148 & -0.028532 & 0.0004148 \\
\hline 3 & 0.0262679 & 0.0001638 & 0.0262679 & 0.0001638 & -0.021903 & 0.0003142 & -0.021903 & 0.0003142 \\
\hline 2 & 0.0133390 & 0.0000967 & 0.0133390 & 0.0000967 & -0.013202 & 0.0002061 & -0.013202 & 0.0002061 \\
\hline 1 & 0.0032673 & 0.0000352 & 0.0032673 & 0.0000352 & -0.004790 & 0.0000842 & -0.004790 & 0.0000842 \\
\hline
\end{tabular}
analysis value.

$\mathrm{X}$ direction earthquake load selection:

$$
0.90 \times 2694.018=2424.617>1913.870 \rightarrow 2424.617 \mathrm{t}
$$

Y direction earthquake load selection:

$$
0.90 \times 2694.018=2424.617>2110.676 \rightarrow 2424.617 \mathrm{t}
$$

Table 3: $\quad$ Maximum displacements $(\mathrm{cm})$. 
Earthquake displacements are found.

Maximum displacements for existing structure are:

$$
\delta \mathrm{x}=0.0383761, \quad \delta \mathrm{y}=0.0285320 \mathrm{t} .
$$
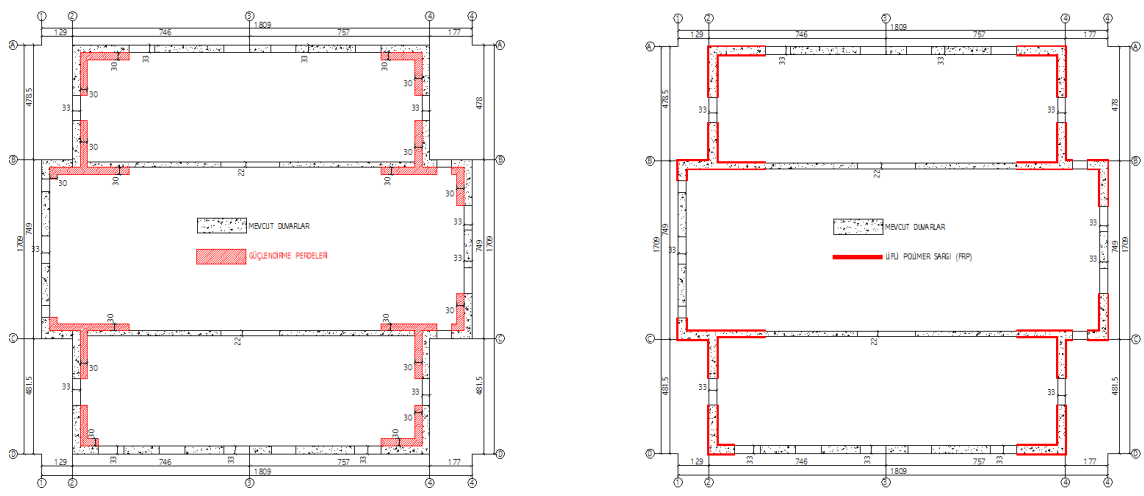

Figure 2: $\quad$ RC and GFRP strengthened structure plan.

\subsection{Analysis of masonry structure strengthened with Glass Fiber Reinforced Polymer (GFRP)}

GFRP is applied to two faces of masonry walls instead of RC walls. GFRP is applied full face on the walls, not diagonally. Properties of GFRP are explained in the following table.

Table 4: $\quad$ GFRP properties.

\begin{tabular}{|c|c|c|c|}
\hline GFRP TYPE & $\begin{array}{c}\text { Tensile } \\
\text { strength } \\
(\mathrm{kg} / \mathrm{cm} 2)\end{array}$ & $\begin{array}{c}\text { Modulus of } \\
\text { elasticity } \\
(\mathrm{kg} / \mathrm{cm} 2)\end{array}$ & $\begin{array}{c}\text { Ultimate } \\
\text { stress (\%) }\end{array}$ \\
\hline \hline MbraceFibre C1-30 & 34300 & 2300000 & 1.5 \\
\hline
\end{tabular}

Equivalent RC cross-section and modulus of elasticity of GFRC and masonry shear wall which are work together are found from breaking load of experiment result which are made by Özsaraç and Torubalc1 [4].

Breaking load of existing masonry wall $=\mathrm{FT}=11.33 \mathrm{kN}$.

Breaking load of GFRP strengthened masonry wall $=\mathrm{FF}=29.33 \mathrm{kN}$.

$$
\begin{gathered}
\mathrm{k} 2=\mathrm{FF} / \mathrm{FT}=29.33 / 11.33=2.59 \\
\mathrm{Eeş2}=\mathrm{ET}+\mathrm{EF} / 2.59=3000+2300000 / 2.59=891000 \mathrm{~kg} / \mathrm{cm}^{2} \\
\text { beş2 }=\mathrm{bT}+\mathrm{bF} / 2.59=33+(0,2 \times 2) / 2.59=34 \mathrm{~cm} .
\end{gathered}
$$

GFRP strengthened structural system is analyzed with using equivalent GFRP cross-section and elasticity coefficient and examined results. 
Spectrum coefficient for strengthened masonry structures $\mathrm{S}(\mathrm{T})=2.5$ and earthquake load reduction coefficient for strengthened masonry system $\operatorname{Ra}(\mathrm{t})=2$

Earthquake loads for $\mathrm{x}$ and $\mathrm{y}$ direction are found

$$
\begin{aligned}
& \mathrm{Vtx}=\mathrm{W} . \mathrm{A}(\mathrm{t}) / \operatorname{Ra}(\mathrm{t})>0,10 \text {. Ao.I.W } 2260.11>180.81 \mathrm{t} \\
& \mathrm{Vty}=\mathrm{W} . \mathrm{A}(\mathrm{t}) / \operatorname{Ra}(\mathrm{t})>0,10 \text {. Ao.I.W } 2260.11>180.81 \mathrm{t}
\end{aligned}
$$

Modal analysis minimum load ratio multiplies with equivalent earthquake loads. Earthquake load select from big one between multiplicand value and modal analysis value.

$\mathrm{X}$ direction earthquake load selection:

$$
0.90 \times 2260.106=2034.096>1627.951 \rightarrow 2034.096 \mathrm{t}
$$

$\mathrm{Y}$ direction earthquake load selection:

$$
0.90 \times 2260.106=2034.096>1702.915 \rightarrow 2034.096 \mathrm{t}
$$

Table 5: $\quad$ Maximum displacements (cm).

\begin{tabular}{|r|c|c|c|c|c|c|c|c|}
\hline \multirow{2}{*}{ Kat } & \multicolumn{2}{|c|}{9. yúkleme } & \multicolumn{2}{|c|}{10. yúkleme } & \multicolumn{2}{|c|}{11. yúkleme } & \multicolumn{2}{|c|}{12. yükleme } \\
\cline { 2 - 9 }$(\mathrm{dyf})$ & $\delta \mathrm{x}(\mathrm{m})$ & $\theta \mathrm{z}(\mathrm{rad})$ & $\delta \mathrm{x}(\mathrm{m})$ & $\theta \mathrm{z}(\mathrm{rad})$ & $\delta \mathrm{y}(\mathrm{m})$ & $\theta \mathrm{z}(\mathrm{rad})$ & $\delta \mathrm{y}(\mathrm{m})$ & $\theta \mathrm{z}$ (rad) \\
\hline 4 & 0.0946083 & 0.0006067 & 0.0946083 & 0.0006067 & -0.062306 & 0.0004859 & -0.062306 & 0.0004859 \\
3 & 0.0645475 & 0.0004544 & 0.0645475 & 0.0004544 & -0.046252 & 0.0003993 & -0.046252 & 0.0003993 \\
2 & 0.0339478 & 0.0002673 & 0.0339478 & 0.0002673 & -0.026059 & 0.0002765 & -0.026059 & 0.0002765 \\
1 & 0.0099263 & 0.0001049 & 0.0099263 & 0.0001049 & -0.008675 & 0.0001222 & -0.008675 & 0.0001222 \\
\hline
\end{tabular}

Maximum displacements are found.

Maximum displacements for existing structure:

$$
\delta \mathrm{x}=0.094608, \delta \mathrm{y}=0.062306 \mathrm{~m} \text {. }
$$

\section{Conclusion}

Consequently, analysis of an existing structure, an RC strengthened structure and a GFRP strengthened structure are compared from the point of earthquake loads and displacements.

Earthquake loads of existing structure

$$
\mathrm{F}_{\mathrm{x}}=\mathrm{F}_{\mathrm{y}}=2033 \mathrm{t}
$$

Earthquake load of RC strengthened structure

$$
\mathrm{F}_{\mathrm{x}}=\mathrm{F}_{\mathrm{y}}=2424 \mathrm{t}
$$

Earthquake load of GFRP strengthened structure

$$
\mathrm{F}_{\mathrm{x}}=\mathrm{F}_{\mathrm{y}}=2034 \mathrm{t}
$$

Maximum displacements compared to three conditions

Maximum displacements of existing structure

$$
\delta x=0.1487, \delta y=0.2583 \mathrm{~m}
$$

Maximum displacements of RC strengthened structure

$$
\delta x=0.0383, \delta y=0.0285 \mathrm{~m}
$$

Maximum displacements of GFRP strengthened structure

$$
\delta x=0.0946, \delta y=0.0623 \mathrm{~m}
$$

Because of the weak wooden structural system of the slabs, large value displacements are found in the analysis.

Maximum displacements are compared in table 6. 

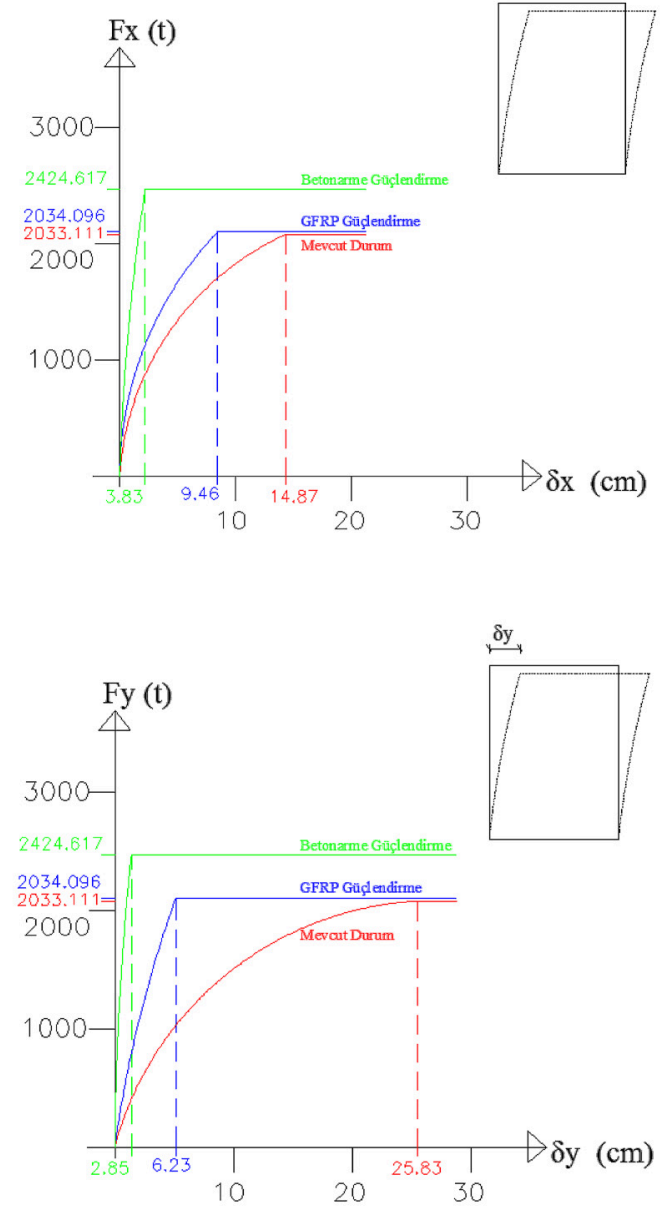

Figure 3: $\quad$ Earthquake loads- $\mathrm{x}$ and y direction displacement curves.

Table 6: $\quad$ Maximum displacements.

\begin{tabular}{|c|c|c|c|}
\hline $\begin{array}{c}\text { Strengthened } \\
\text { System }\end{array}$ & $\begin{array}{c}\text { Existing } \\
\text { structure }(\mathrm{cm})\end{array}$ & $\begin{array}{c}\text { RC } \\
\text { strengthened } \\
\text { structure }(\mathrm{cm})\end{array}$ & $\begin{array}{c}\text { GFRP } \\
\text { strengthened } \\
\text { structure }(\mathrm{cm})\end{array}$ \\
\hline \hline$\delta \mathrm{x}$ & 14.87 & 3.83 & 9.46 \\
\hline$\delta \mathrm{y}$ & 25.83 & 2.85 & 6.23 \\
\hline
\end{tabular}


Earthquake loads and maximum displacements for $\mathrm{x}$ and $\mathrm{y}$ directions are displayed on graphical representations

The analyses show the displacements of the strengthened systems are reduced, and these are improved 9 and 4 times according to the present building respectively. Finally, RC shear walls strengthened system provides new code criteria. However, if it is necessary to keep outdoor views and for easy application, the FRP/GFRP grid bonded strengthened systems are recommended instead of the additional RC shear wall system.

\section{References}

[1] Torunbalcı N, İsler O. Performance of R/C and Masonry Structures During the 2003 Bingöl Earthquake in Turkey', 13 ${ }^{\text {th }}$ World Conference on Earthquake Engineering,, Vancouver, B.C., Canada, August 1-6, 2004, Paper No. 3145.

[2] DBYBHY. 2007 Earthquake Code of Turkey, Bayındırlık ve İskan Bakanlığı, Ankara.

[3] Franklin, S., Lynch J., Abrams D, 2001. Performance of Rehabilitated URM Shear Walls: Flexural Behaviour of Piers, Department of Civil Engineering, University of Illinois at Urbana-Champaign, Illinois.

[4] Özsaraç, S., Torubalc1, N. 2008. Experimental study of GFRP strengthened of load-carrying wall at masonry structures, Istanbul Technical University. 